\title{
Kinderrechte in der Pandemie
}

\author{
Raus aus der Krise, rein ins Grundgesetz
}

Die Pandemie lässt uns seit zwei Jahren schonungslos auf die Mangellagen Deutschlands blicken. Die Maßnahmen zum Infektionsschutz potenzieren Kinderarmut, soziale Ungleichheit und problematische Kinderschutzverläufe. Die Berücksichtigung der Belange und Perspektiven von Kindern und Jugendlichen bleibt hier weit hinter den Vorgaben der UN-Kinderrechtskonvention zurück.

T rotz des bevorstehenden 30-jährigen Jubiläums der UN-Kinderrechtskonvention (UN-KRK) in Deutschland sind die Rechte der Kinder im anhaltenden Pandemiegeschehen weitgehend unbeachtet geblieben. Der von der UN-KRK geforderte Paradigmenwechsel, Kinder ${ }^{1}$ als Rechtssubjekte anzuerkennen, scheint auch nach 30 Jahren nur schwer Eingang in die politische Praxis und in das gesellschaftliche Bewusstsein gefunden zu haben. Am Beispiel der Pandemiebekämpfung möchte dieser Beitrag die Notwendigkeit der Beachtung und Beteiligung von Kindern im Sinne der Vorgaben der UN-KRK und die Forderung nach einer Verankerung der Kinderrechte im Grundgesetz miteinander in Bezug setzen.

Im Folgenden wird die Ausgangslage mit Fokus auf die Sichtbarkeit und Rechtssubjektstellung von Kindern und Jugendlichen in der Pandemie dargestellt. Im Anschluss daran werden die Anwendbarkeit, Vorgaben und Reichweite der UN-KRK als völkerrechtlicher Ver-

\author{
Marie Nadjafi-Bösch \\ Berlin, Deutschland \\ Referentin Koordinierungsstelle Kinderrechte, Deutsches \\ Kinderhilfswerk \\ nadjafi@dkhw.de
}

Zusammenfassung Die Bedarfe und Interessen von Kindern als eigene Rechtssubjekte, scheinen während der Maßnahmen zur Bekämpfung der Pandemie in einer Entität von Kindern und Erwachsenen unterzugehen und bleiben auch 30 Jahre nach der Geltung der UN-Kinderrechtskonvention hinter deren Vorgaben zurück.

Schlüsselwörter COVID-19, Pandemie, Armut, UNKinderrechte, Kindeswohlvorrang, Rechtssubjekte, Beteiligung, Perspektive, Grundgesetz trag für Deutschland als Vertragsstaat skizziert. Dabei werden die Umsetzungsdefizite des Prinzips des Kindeswohlvorranges nach Art. 3 Abs. 1 UN-KRK und das damit eng verknüpfte Beteiligungsrecht gemäß Art. 12 Abs. 1 UN-KRK im Kontext der Pandemie aufgezeigt. Abschließend werden einige Kernargumente der Forderung Kinderrechte ins Grundgesetz abgebildet und unter Bezugnahme auf den emanzipatorischen Charakter der Kinderrechte diskutiert.

\section{Kinder und Jugendliche in der Krise}

Dass die Corona-Pandemie und die Maßnahmen zu deren Eindämmung nicht auf alle Alters- und Sozialgruppen gleich wirk $(t) e n$, scheint inzwischen eine Binsenweisheit. Für Kinder und vor allem für armutsbetroffene Minderjährige bedeuteten die Corona-Maßnahmen und insbesondere der so genannte Lockdown enorme Einschränkungen ihrer zivilen, sozialen und kulturellen Rechte auf Schutz, Förderung und Beteiligung besonders in den Bereichen Kindeswohl, Gesundheit und Bildung (Klundt 2021). Die viel benutzte Brennglas-Metapher, welche verbildlicht, wie die Maßnahmen zur Bekämpfung der Pandemie bereits vorhandene Ungleichheiten potenziert, kann nahezu für alle gesellschaftlichen und sozialen Kontexte beispielhaft angeführt werden. Die Krise legt bestehende Versorgungslücken und Mängel im Sozial-, Sicherungs- und Bildungssystem schonungslos offen und verschärft sie. So gerieten armutsbetroffene Familien beispielsweise durch die fehlende Mittagsverpflegung in Schulen und Kitas sowie krisenbedingte Mehrkosten besonders in Bedrängnis.

Auch wurde die Mangellage in der Kinder- und Jugendhilfe in der Pandemie deutlicher denn je (DKHW 2020). Der Umstand, dass Kinderschutz noch immer keine Chef_ innen-Sache ist, hat in der Krise besondere Auswirkun- 


\section{Durchblick: Kinderrechte in Corona-Zeiten}

gen auf armutsbetroffene Kinder, welche aufgrund der Maßnahmen zur Corona-Eindämmung spezifische Ausmaße erreicht haben. Die Folgen der seit Jahren von Praxis und Forschung monierten Anbindung der finanziellen und personellen Ausstattung der Jugendämter an unterster anstatt föderaler oberster Ebene, wurden während der Maßnahmen zur Bekämpfung von COVID-19 in all ihrer Härte sichtbar (Beckmann und Berneiser 2021). Ebenso verschärfte sich die Situation von vielen geflüchteten Kindern und ihren Familien durch die Pandemiemaßnahmen. Dass das Konzept der zentralen Unterbringung geflüchteter Familien und Kinder in Anker- und funktionsgleichen Einrichtungen eine Vielzahl von Risiken für Kinder birgt, zeigt sich in der Pandemie deutlich. Diese Orte der Ausgrenzung, Stigmatisierung und Isolation entwickelten sich jüngst aufgrund der meist räumlichen Abgeschiedenheit und einer gewissen Geschlossenheit zu sogenannten Corona-Hotspots, die gesundheitliche und psychosoziale Belastungen bei Kindern und ihren Familien verursachen (Pro Asyl 2021). Während der Pandemie sind junge Menschen aufgrund zunehmend psychischer Belastungen einer Dauer-Stress-Situation ausgesetzt. Mittlerweile macht eine Vielzahl von Untersuchungen deutlich, dass junge Menschen von den Einschränkungen massiv belastet sind. Die Mahnung der Kinderkommission des Deutschen Bundestages aus dem Jahr 2020, dass jede politische Entscheidung zur Bekämpfung der Corona-Pandemie mit dem Blick auf Kinder und Jugendliche eine besondere Interessenabwägung erfahren muss, scheint bisher ins Leere zu laufen (Pressemitteilung der Kommission zur Wahrnehmung der Belange der Kinder 2020). Die öffentlichen Debatten wurden sehr stark aus der Perspektive von Erwachsenen geführt. Dabei fehlt eine zentrale Perspektive - die der Kinder (Deutscher Bundestag 2020). Auch in der gegenwärtigen sogenannten dritten Phase des Pandemiegeschehens befinden sich Familien und v. a. Kinder in einer Art Chaos-Situation (Baumann 2022). So fehlt es beispielsweise weiterhin an Infrastrukturen zum Infektionsschutz in Schulen und Einrichtungen der Kinder- und Jugendhilfe. Die Dimensionen der psychischen Belastungen und Folgeerscheinungen für Kinder und Jugendliche sind noch nicht abzusehen.

\section{Mangelnde Sichtbarkeit von Kindern als eigene Rechtssubjekte}

Bereits nach einem Jahr Covid-19-Pandemie zeichnete sich ab, dass Kinder und Jugendliche als Träger_innen eigener Rechte in den öffentlichen Beobachtungsschemata von Politik und Medien weitestgehend ausgeblendet werden. Während der Ausgangsbeschränkungen im Frühjahr 2020 wurde zwar durchaus die Sorge um steigende Gewaltraten in Familien diskutiert, Hauptthemen der öffentlichen Debatten bildeten jedoch die Rolle von Kindern und Jugendlichen bzw. Schulen am Infektionsgeschehen, die bildungsökonomischen Konsequenzen der Schließungen von Bildungs- und Betreuungseinrichtungen und die Vereinbarkeit von elterlicher Arbeitswelt und Familien, bzw. Lohnarbeit und Care-Aufgaben. Kinder und Jugendliche erscheinen dabei eher als Organisationsproblem erwachsener Lebenswelten, selbst wenn sich seit Anfang 2021 vermehrt Medienberichte zu den psychischen und medizinischen Langzeitfolgen der Ausgangsbeschränkungen für Kinder und Jugendliche finden lassen (Albert und Marke 2021).

Auch das aktuelle Fazit zum forschungspraktischen Mitspracherecht von Kindern und Jugendlichen macht deutlich, dass Kinder als Akteure weitestgehend ausgeblendet werden (Albert und Marke 2021). Die Beteiligung von Kindern an der Forschung und am Forschungsdesign selbst fällt gering aus (vgl. u.a. Bertelsmann 2020). Der Großteil der Informationen zu der Lebenswelt von Kindern wurde bislang häufig von Eltern aus der Mittelschicht erlangt, welche als Gatekeeper zum Zugang zum Wissen über Kinder und Jugendliche anzusehen sind (Albert und Marke 2021). Entsprechend dieser Erwachsenen-Zentriertheit liegen die Schwerpunktthemen im Kontext der Diskussion um die Bedürfnisse von Kindern und Jugendlichen und der vorherrschenden Kinderpolitik, beispielsweise beim Schulerfolg und Medienkonsum (ebenda). Aus einer den Kinderrechten verpflichteten Position ergeben sich Wissenslücken hinsichtlich der Themen Schutz vor Gewalt (Art. 19 UN-KRK), Vorrang des Kindeswohls (Art. 3 Abs. 1 UN-KRK), Recht auf Bildung, Art. (28 UN-KRK) Recht auf Gesundheit (Art. 24 UNKRK) und Recht auf Beteiligung (Art. 12 UN-KRK) (Albert und Marke 2021).

Dabei wird gerade in der Krise deutlich, wie dringend geboten es ist, sich gesamtgesellschaftlich dieser Errungenschaft und Anforderung der UN-KRK zugunsten Kindern und Jugendlichen gewahr zu werden. Das Kind wird mithin als eigenständiges Rechtssubjekt anerkannt, dem seine Rechte auch in Krisenzeiten unveräußerlich zustehen, ebenso wie Erwachsenen. Ähnlich wie die Behindertenrechtskonvention (UN-BRK) und Frauenrechtskonvention (UN-FRK) ist die UN-KRK aufgrund ihrer Genese als internationale Antidiskriminierungsmaßnahme der menschenrechtlichen Emanzipation zu verstehen (Schmahl 2017). Schutz und Rechte werden dem Kind um seiner selbst willen garantiert, es wird nicht mehr nur als Teil der Familie oder einer sozialen Gruppe wahrgenommen (Schmahl 2017). Da Kinder und Jugendliche allerdings bei der Durchsetzung ihrer Rechte auf Erwachsene angewiesen sind, gibt die Konvention den Vertragsstaaten klare Vorgaben, um die Rechte des Kindes auch zur An- 
wendung zu bringen. Dabei formuliert die Konvention ein klares Pflichtenprogramm, welches, gemeinsam mit den einzelnen im Vertragstext verbürgten Rechten, 30 Jahre nach Ratifikation auch zumindest aus rechtsdogmatischer Sicht unbestritten unmittelbare Anwendung in Deutschland findet. Dazu wird im Folgenden die unmittelbare Anwendungs- und Umsetzungspflicht in Bezug auf den Kindeswohlvorrang (Art. 3 Abs. 1 UN-KRK) und das damit eng verknüpfte Recht auf Beteiligung von Kindern und Jugendlichen (Art. 12 Abs. 1 UN-KRK) in Deutschland skizziert.

\section{Kindeswohl: Nur durch Beteiligung umfassend festzustellen}

Völkerrechtliche Verträge wie die UN-KRK haben in Deutschland über das deutsche Grundgesetz (Art. 59 Abs. 2, S. 1 GG) den Rang eines einfachen Bundesgesetzes. Dementsprechend genießt die UN-KRK Geltungsvorrang vor dem Landesrecht (Art. 31 GG). Darüber hinaus müssen die Bestimmungen des Vertrages mit deutschen Bundesgesetzen methodisch vertretbar ausgelegt werden (Schmahl 2017). Der Regelungsgehalt und die Reichweite der völkerrechtlichen Umsetzungspflicht für den Vertragsstaat Deutschland bemisst sich nach Art. 4 UN-KRK. Demnach müssen die Vertragsstaaten alle geeigneten Gesetzgebungs-, Verwaltungs- und sonstige Maßnahmen treffen.

Wie der Kinderrechtsausschusses (KRA) in seinen allgemeinen Bemerkungen, die regelmäßig als Leitlinien und Auslegungshilfe zur Umsetzung der Kinderrechte heranzuziehen sind, feststellt, erfordert Art. 4 UN-KRK nationale Strategien und Aktionspläne unter differenzierter Betrachtung von Kindern in Bezug auf Status, Alter und Geschlecht zur praxisnahen Durchsetzung der Konventionsrechte. Die föderalen Kompetenzverteilungen und Strukturen stellen Deutschland dabei nicht von den konventionsrechtlichen Verpflichtungen frei (Art. 27 Wiener Vertragsrechtskonvention), sondern müssen entsprechend koordiniert werden. Deutschland ist als Vertragsstaat zur Durchsetzung der Kinderrechte nicht auf den Erlass legislativer oder administrativer Maßnahmen beschränkt. Es sind alle erdenklichen Maßnahmen zu ergreifen, und zwar justizielle, wirtschaftliche oder soziale Maßnahmen (Allgemeinen Bemerkungen Kinderrechteausschuss , UN, Committee on the Rights of the Child 2016).

\section{Das Prinzip des Kindeswohlvorranges: zwischen Anspruch und Wirklichkeit}

Eine besondere Stellung kommt dem Kindeswohlprinzip nach Art. 3 Abs. 1 UN-KRK zu. Demnach ist das Kindeswohl bei allen Maßnahmen, die das Kind betreffen - gleich, ob sie von öffentlichen oder privaten Einrichtun- gen der sozialen Fürsorge, Gerichten, Verwaltungsbehörden oder Gesetzgebungsorganen getroffen werden - ein Gesichtspunkt, der vorrangig zu berücksichtigen ist. Bei der Berücksichtigung des Kindeswohls nach Art. 3 Abs. 1 KRK geht es darum, dass sich die Entscheidungsträger_innen über die Auswirkungen der jeweiligen Entscheidung auf ein individuelles Kind, eine Gruppe von Kindern oder Kinder im Allgemeinen bewusst werden. Das Kindeswohl soll damit nicht eine Entscheidung vorgeben, sondern als eine wesentliche Leitlinie fungieren (Lorz und Sauer 2011). Bei der Definition des Kindeswohls bzw. der Festlegung des Wohles des Kindes ist die Perspektive und die Ansicht des betroffenen Kindes oder der Gruppe der Kinder dem Entwicklungsstand angemessen zu berücksichtigen. Das Beteiligungsrecht gibt die Methode zur Festlegung des Kindeswohls vor (Schmahl 2017). Vor diesem Hintergrund ist gegenwärtig nicht festzustellen, dass der Kindeswohlvorrang sich als Grundprinzip durch die politischen Entscheidungen von Bund und Ländern gezogen hätte. Auch wurden Kinder und Jugendliche nicht an Entscheidungsprozessen beteiligt (Deutscher Bundestag, 2020). Der Schutz und die besondere Perspektive von Kindern wurden bislang nicht in die Pandemiepläne des Bundes und der Länder aufgenommen. Entsprechend wenig Gehör erfuhren Kinder- und Jugendorganisationen bei den staatlichen Grundsatzentscheidungen zur Bekämpfung der Covid-19-Pandemie (Feige und Gerbig 2020, S. 5). Letztlich ist angesichts bestehender Möglichkeiten, welche die UN-KRK zugunsten der Einbeziehung der Kindeswohlprüfung unter Beteiligung von Kindern und Jugendlichen bietet, zu fragen, wie es gelingen kann, den Rechten der Kinder und Jugendlichen in Deutschland zur Um- und Durchsetzung zu verhelfen und dies besonders in Krisensituationen. Dabei ist die Forderung nach der Verankerung der Kinderrechte im Grundgesetz besonders unter Beachtung des emanzipatorischen Erfordernisses, Kinder nicht nur als Teil der Familie, sondern als eigenständiges Rechtssubjekt zu betrachten, in den Blick zu nehmen.

\section{Kinderrechte ins Grundgesetz - gerade jetzt!}

Das Vorhaben, die Kinderrechte ins Grundgesetz aufzunehmen, wurde von einem breiten zivilgesellschaftlichen Bündnis, zuletzt in einer gemeinsamen Initiative von über 100 Organisationen eingefordert. Aufgrund einer Vielzahl von Divergenzen scheiterte das Vorhaben (Deutsches Kinderhilfswerk 2022). Inzwischen hat sich die neue Bundesregierung die Verankerung der Kinderrechte im Grundgesetz vorgenommen. Es bleibt abzuwarten, ob nunmehr eine Formulierung gefunden werden kann, welche den Vorgaben der UN-KRK entspricht. In der Pandemie zeigt sich nun an den Entscheidungen 


\section{Durchblick: Kinderrechte in Corona-Zeiten}

von Bund, Ländern und Kommunen in aller Deutlichkeit, dass die besondere Bedeutung des Kindeswohlprinzips (Art. 3 Abs. 1 UN-KRK) und ihre unmittelbare Anwendbarkeit ohne eine explizite Grundrechtsposition in der deutschen Verfassung vielfach verkannt wird. Nur durch starke Kinderrechte im Grundgesetz kann das Wohl des Kindes bei staatlichen Entscheidungen ein größeres Gewicht bekommen. Besonders in Deutschland scheint der historisch gewachsene und viel diskutierte nationale Kindeswohlbegriff dazu zu führen, dass die Rechtsstellung des Kindes sehr eng mit dem Kinderschutzgedanken verknüpft ist und wenig Raum für die Betrachtung des Kindes als eigenes Rechtssubjekt mit eigenen Interessen lässt, anders z. B. im angelsächsischen Bereich, wo die Bezeichnung „best interest of the child“ zuvörderst auf das Interesse von Kindern abstellt (Nadjafi-Bösch 2022).

Durch die Verankerung der Kinderrechte, insbesondere des Kindeswohlprinzips nach Art. 3 Abs. 1 UNKRK, könnte der eingeforderte Perspektivwechsel und der emanzipatorische Charakter der UN-Kinderrechte befeuert werden. Mit Verweis auf die Soziologin Alanen soll hier auf die Parallelitäten bezüglich der sozialen Situation von Frauen und Kindern hingewiesen werden. Die Beziehung von Kindern und Erwachsenen ist im Sinne einer Analogie zu der Beziehung zwischen Mann und Frau, aus einer patriarchalen Sichtweise, gleichzusetzen (Alanen 1994). Durch die Erwachsenenzentriertheit können die Interessen von Kindern und Jugendlichen schnell hinter den Interessen dieser zurücktreten und letztlich unsichtbar werden. Eine Aufnahme der Kinderrechte ins Grundgesetz hätte zur Folge, dass, analog zum Gleichstellungsauftrag des Art. 3 GG, dem Kindeswohl auf Bundes- und Landesebene durch institutionelle und strukturelle Verankerungen zur Beachtung und Umsetzung verholfen werden könnte.

\section{Lehrreiches aus der Krise}

30 Jahre nach der Ratifikation der Kinderrechte in Deutschland ist der gesamtgesellschaftliche, politische und mediale Umgang mit den Kindern und Jugendlichen in der Pandemie zum Anlass zu nehmen, die Notwendigkeit der Verankerung der Kinderrechte, entsprechend ihren Vorgaben, ins Grundgesetz anzuerkennen. Die letzten zwei Jahre haben in aller Deutlichkeit gezeigt, dass die Umsetzung der Kinderrechte auf Seiten von Verwaltung, Gesetzgeber und Rechtsprechung auf allen Ebenen Defizite aufweist und die Belange und Perspektiven von Kindern aus dem Blick geraten.
Angenommen. 3. Februar 2022

1. Im Sinne der UN-KRK ist ein Kind jeder Mensch, der das achtzehnte Lebensjahr noch nicht vollendet hat, soweit die Volljährigkeit nach dem auf das Kind anzuwendenden Recht nicht früher eintritt, vgl. Art. 1 UN.-KRK.

\section{Literatur}

Alanen, L. (1994). Gender and generation: feminism and the "child question”. In J. Qvortrup, M. Bardy, G. Sgritta \& H. Wintersberger (Hrsg.), Childhood matters. Social Theorie, practice and politics (S. 27-42). Aldershot: Avebury.

Albert, L., \& Marke, V. (2021). Stimme der Kinder oder Stimmung in der Familie? ZSE, Zeitschrift für Soziologie der Erziehung und Sozialisation, 2/2021, 228-233.

Baumann, M. (2022). Familien im Corona Stress. „Zum dritten Mal in einer Chaos-Situation “, Tagesschau-Interview vom 23.01.2022. https://www. tagesschau.de/inland/gesellschaft/interview-omikron-schulen-kitas-101. html. Zugegriffen: 31. Jan. 2022.

Beckmann, K., \& Berneiser, C. (2021). Kinderschutz in der (Corona-) Krise: Zwischen Anspruch und Wirklichkeit, Heft 1, 2021 ZKJ, Zeitschrift für Kindschaftsrecht und Jugendhilfe, S. 4-10. https://www.reguvis. de/fileadmin/FamSoz-Portal/Dokumente/Beitraege/Beckmann-Berneiser_ ZKJ_01_2021.pdf. Zugegriffen: 31. Jan. 2022.

Bertelsmann Stiftung (Hrsg.). (2020). Factsheet Kinderarmut in Deutschland. (Funcke, A./Menne, S.). Gütersloh. https://www.bertelsmann-stiftung. de/fileadmin/files/BSt/Publikationen/GrauePublikationen/291_2020_BST_ Facsheet_Kinderarmut_SGB-II_Daten_ID967.pdf. Zugegriffen: 31. Jan. 2022

Deutscher Bundestag (2020). https://dserver.bundestag.de/ btd/19/191/1919146.pdf. Zugegriffen: 27. Feb. 2022.

Deutsches Kinderhilfswerk e. V. (Hrsg.). (2020). Kinderrechte in Zeiten von Corona wichtiger denn je! https://www.dkhw.de/fileadmin/Redaktion/1_Unsere_Arbeit/1_Schwerpunkte/2_Kinderrechte/2.3_Kinderrechte_in Deutschland/Positionspapier_Kinderrechte_Corona.pdf. Zugegriffen: 31 . Jan. 2022.

Deutsches Kinderhilfswerk e. V. (Hrsg.). (2021). Kinderrechte ins Grundgesetz aber richtig, Aktionsbünndis Kinderrechte. https://www.dkhw.de/ fileadmin/Redaktion/1_Unsere_Arbeit/1_Schwerpunkte/2_Kinderrechte/2.17_Kinderrechte_ins_Grundgesetz/Appell_Kinderrechte_ins_Grundgesetz_-_aber_richtig_17-05-21.pdf. Zugegriffen: 31. Jan. $202 \overline{2}$.

Deutsches Kinderhilfswerk e. V. (Hrsg.). (2022). Kinderrechte ins Grundgesetz; Aktionsbündnis und Inititaive, vgl. https://www.dkhw.de/schwerpunkte/kinderrechte/kinderrechte-ins-grundgesetz/?gclid=Cj0KCQiA3yQBhD3ARIsAHuHT66q5D1enmj-gORJegnBaK3nbB2Qavcd6Qysqd2yrDxZuuQL6YQIUnkaAmPkEALw_wcB. Zugegriffen: 27. Feb. 2022.

Feige, J. und Gerbig, S (2020). Kinderrechte in Zeiten der Corona Pandemie, Stellungnahme 2020, Deutsches Institut für Menschenrechte, Monitoring-Stelle UN-Kinderrechtskonvention (Hrsg.). https://nbn-resolving.org/ urn:nbn:de:0168-ssoar-73103-4. Zugegriffen: 27. Feb. 2022.

General Comment, Convention on the Rights of the Child, No. 19 (2016). On public budgeting for the realiziation of the children's rights (art. 4), United Nations Human Rights Treaty Bodies, databse, CRC/C/GC/19, 20.06.2016, Rn. 21. https://tbinternet.ohchr.org/_layouts/15/treatybodyexternal/Download.aspx? symbolno=CRC $\% 2$ FC $\% 2$ FGC $\% 2$ F $19 \&$ Lang=en. Zugegriffen: 31. Jan. 2022.

Klundt, M. (2021). Soziale Spaltung und Corona-Kapitalismus. Sozial Extra, 45, 13-18. https://doi.org/10.1007/s12054-020-00343-x.

Kommission zur Wahrnehmung der Belange der Kinder. Pressemitteilung vom 7. Mai 2020. https://www.bundestag.de/resource/blob/694638/ac02d6edf056ef6bd6d111385fe1637d/Situation-der-Kinder-in-der-Coronapandemie-data.pdf. Zugegriffen: 31. Jan. 2022.

Lorz, A., \& Sauer, A. (2011). Kinderrechte ohne Vorbehalt, Die Folgen der unmittelbaren Anwendbarkeit des Kindeswohlsvorrangs nach der UN-Kinderrechtskonvention in der deutschen Rechtsordnung. MRM, Menschenrechts Magazin, 2/2011, 5-16. 
Nadjafi-Bösch, M. (2022). 30 Jahre Ratifizierung der UN-Kinderrechte. Wie steht es um das Prinzip des Kindeswohlvorranges? Zum Umsetzungsstand in Deutschland am Beispiel kindgerechter Justiz. MRM, Menschenrechts Magazin, 1/2022, Verlag. Publikation im April 22.

Pro Asyl e. V. (2021). Bedeutet unser Leben nichts? Erfahrungen von Asylsuchenden in Flüchtlingsunterkünften während der Corona-Pandemie. (Huke, Nikolai). https://www.proasyl.de/wp-content/uploads/210809_PA_ Lager.pdf. Zugegriffen: 31. Jan. 2022.

Schmahl, S. (2017). Kinderrechtskonvention mit Zusatzprotokollen, Handkommentar (2. Aufl.). Baden-Baden: Nomos.

UN, Committee on the Rights of the Child (2016). General comment No. 19 on public budgeting for the realization of children's rights (art. 4), UN Doc. CRC/C/GC/19, 2016, S. 7, vgl. https://digitallibrary.un.org/record/838730. Zugegriffen: 27. Feb. 2022.

Hier steht eine Anzeige. Springer 